\title{
Estudio de las propiedades anticorrosivas sobre acero SAE 1010 de extractos acuosos de romero (Rosmarinus officinalis), laurel (Laurus nobilis) y falso incienso (Plectranthus coleoides)
}

\author{
Study of the anticorrosive properties on SAE \\ 1010 steel of rosemary (Rosmarinus officinalis), \\ laurel (Laurus nobilis) and false incense (Plectranthus coleoides)
}

Christian Byrne $^{1,2}$, Martín Ramírez ${ }^{3}$, Ezequiel Di Santo ${ }^{3}$, Nicol Cristiano $^{3}$, Cecilia Deyá ${ }^{1,4}$, Oriana D’Alessandro ${ }^{1,2}$

\author{
${ }^{1}$ CIDEPINT-CICPBA- CONICET-Facultad de Ingeniería-UNLP, Av. 52 e/121 y 122, (B1900AYB) La Plata, Buenos \\ Aires, Argentina \\ ${ }^{2}$ Facultad de Ciencias Exactas-UNLP, 47 y 115 s/n (B1900AJK), La Plata, Buenos Aires, Argentina. \\ ${ }^{3}$ Facultad Regional La Plata-UTN, Av.60 esq. 124 s/n, Berisso, Buenos Aires, Argentina. \\ ${ }^{4}$ Facultad de Ingeniería-UNLP, 1 y 47 s/n (B1900TAG), La Plata, Buenos Aires, Argentina. \\ e-mail: o.dalessandro@ cidepint.ing.unlp.edu.ar
}

\section{RESUMEN}

Las hojas de las plantas perennes Rosmarinus officinalis, Laurus nobilis y Plectranthus coleoides presentan una gran cantidad de compuestos químicos que contienen heteroátomos en su estructura. Diversos estudios han reportado que este tipo de heterocompuestos presentan una gran afinidad química por las superficies metálicas. En este trabajo se presenta la obtención, caracterización y estudio del desempeño anticorrosivo sobre acero SAE 1010 de los extractos acuosos de las hojas de las plantas perennes antes mencionadas. Las especies vegetales fueron cultivadas y cosechadas en la zona del Gran La Plata, Provincia de Buenos Aires, República Argentina. La preparación de los extractos acuosos se realizó mediante la técnica de maceración. El residuo sólido de los extractos acuosos se caracterizó mediante espectroscopia infrarroja con transformada de Fourier (FTIR). La evaluación electroquímica se llevó a cabo mediante la exposición de muestras de acero SAE 1010 durante $24 \mathrm{~h}$ a un medio corrosivo en presencia de cada uno de los extractos, y el posterior análisis de las superficies obtenidas a través de microscopía electrónica de barrido (SEM) y espectroscopia de dispersión de rayos X (EDX). Los resultados fueron comparados con los obtenidos utilizando hidrato de polifosfato de zinc y aluminio (ZAPP), un pigmento anticorrosivo comercial. Los resultados obtenidos permiten concluir que los extractos de las tres especies vegetales inhiben la corrosión del acero SAE 1010 de manera comparable a la efectuada por ZAPP, siendo el extracto de Plectranthus coleoides el que presenta la mejor acción anticorrosiva.

Palabras clave: Acero SAE 1010, Rosmarinus officinalis, Laurus nobilis, Plectranthus coleoides, Extractos vegetales

\section{ABSTRACT}

The leaves of perennials plants Rosmarinus officinalis, Laurus nobilis and Plectranthus coleoides have a large number of chemical compounds that contain heteroatoms in their structure. This type of heterocompounds has been reported to have chemical affinity for metal surfaces. This work presents the obtaining, characterization and study of the anticorrosive performance on SAE 1010 steel of the aqueous extracts of the aforementioned perennials plants. The plant species were cultivated and harvested in the Greater La Plata area, Province of Buenos Aires, Argentine Republic. The preparation of the aqueous extracts 
was carried out using the maceration technique. The solid residue of the aqueous extracts was characterized by Fourier transform infrared spectroscopy (FTIR). Electrochemical evaluation was accomplished by linear polarization tests. The anticorrosive performance was determined by exposing SAE 1010 steel samples for 24 $\mathrm{h}$ to a corrosive medium in the presence of each of the extracts, and the subsequent analysis of the surfaces obtained through scanning electron microscopy (SEM) and X-ray scattering spectroscopy (EDX). The results were compared with those obtained using zinc aluminum polyphosphate hydrate (ZAPP), a commercial anticorrosive pigment. The results obtained allow us to conclude that the extracts of the three plants inhibit the corrosion of SAE 1010 steel in a manner comparable to that carried out by ZAPP, with the extract of Plectranthus coleoides being the one that presents the best anticorrosive action.

Keywords: SAE 1010 steel, Rosmarinus officinalis, Laurus nobilis, Plectranthus coleoides, vegetable extracts.

\section{INTRODUCCIÓN}

En la actualidad, existe un gran interés por encontrar inhibidores de la corrosión no contaminantes que luego puedan ser incorporados en pinturas, con el objetivo de proteger superficies metálicas. Estos inhibidores deben reemplazar a los tradicionales cromatos y fosfatos, ampliamente cuestionados por sus efectos sobre la salud y el medio ambiente. Los primeros han sido objetados debido a que el cromo hexavalente causa una gran variedad de enfermedades, desde dermatitis hasta carcinomas bronquiales [1] y los segundos por causar el aumento de los suministros de fósforo en los ecosistemas acuáticos, degradando los recursos hídricos por eutrofización [2]. Numerosas investigaciones tienden al reemplazo de estos inhibidores por otros amigables con el medioambiente, entre ellos se pueden citar aquellos a base de: taninos [3, 4], zeolitas [5, 6] y aminoácidos [7, 8].

Las plantas comúnmente denominadas "aromáticas" han sido muy utilizadas en actividades asociadas a la gastronomía y a la dispensación fitoterapéutica, debido a los compuestos naturales volátiles que en ellas se presentan $[9,10]$. La composición química de los aceites esenciales depende no sólo de las especies de plantas sino también de su edad, variedad, la parte recolectada, origen, clima, suelo, agroquímicos utilizados, tiempo de almacenamiento y preparación entre otros factores [11].

Los aceites esenciales de estos vegetales son una mezcla compleja de varios componentes. Los compuestos mayoritarios presentes en Rosmarinus officinalis son alcanfor, $\beta$-cariofileno y 1,8-cineol [12, 13], en Laurus nobilis son 1,8-cineol y acetato de $\alpha$-terpenilo $[12,14,15]$ y en Plectranthus coleoides son carvacrol y $\beta$-cariofileno [16-18]. Estos compuestos orgánicos son derivados de los terpenos y poseen heteroátomos o sistemas $\pi$ conjugados en su estructura, lo que les proporciona una elevada afinidad por las superficies metálicas. Al adsorberse sobre el sustrato metálico dan lugar a la formación de películas que dificultan la difusión de iones agresivos como el $\mathrm{Cl}^{-}$hacia la superficie, enlenteciendo significativamente el proceso de corrosión [19, 20].

La maceración es una técnica antigua para la obtención de extractos de compuestos naturales. Si bien no requiere un equipamiento sofisticado, la misma consta de varios pasos: la molienda (para aumentar el área de contacto), el agregado de solvente y la filtración. Para mejorar el proceso difusional se recomienda agitar la mezcla [21]. Mediante esta técnica, utilizando agua como solvente, se pueden obtener antocianinas, taninos, saponinas y terpenoides [22]. Los extractos acuosos derivados de hierbas aromáticas son una alternativa muy interesante debido a que los fitocompuestos se encuentran solubles en agua, lo cual resulta de gran utilidad para realizar los ensayos de corrosión respetando el medioambiente.

En este trabajo se presenta la obtención, caracterización y estudio del desempeño anticorrosivo sobre acero SAE 1010 de extractos acuosos de romero (Rosmarinus officinalis), laurel (Laurus nobilis) y falso incienso (Plectranthus coleoides). Los resultados fueron comparados con los obtenidos utilizando el pigmento HEUCOPHOS ${ }^{\circledR}$ ZAPP (hidrato de polifosfato de zinc y aluminio), un fosfato de tercera generación [23].

\section{MATERIALES Y MÉTODOS}

\subsection{Cosecha de las especies vegetales seleccionadas}

Las especies vegetales seleccionadas (Figura 1) tienen buen crecimiento y una amplia distribución en la zona del Gran La Plata. La cosecha de las partes aéreas de estas hierbas fue realizada durante los meses de marzo y abril de 2019. Luego se seleccionaron sólo las hojas y se secaron en estufa a $40{ }^{\circ} \mathrm{C}$ durante una semana. 


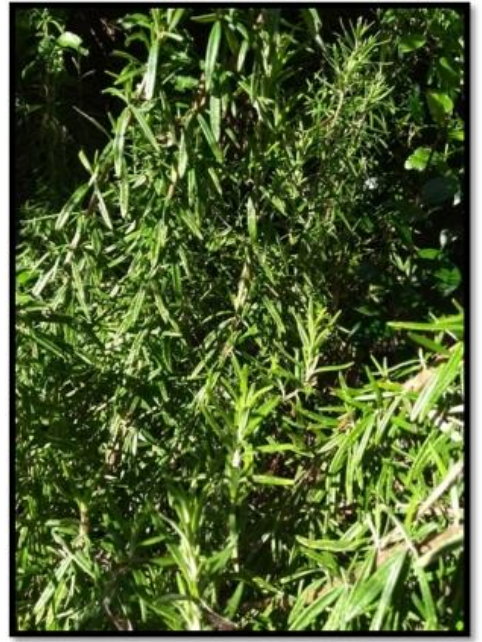

(a)

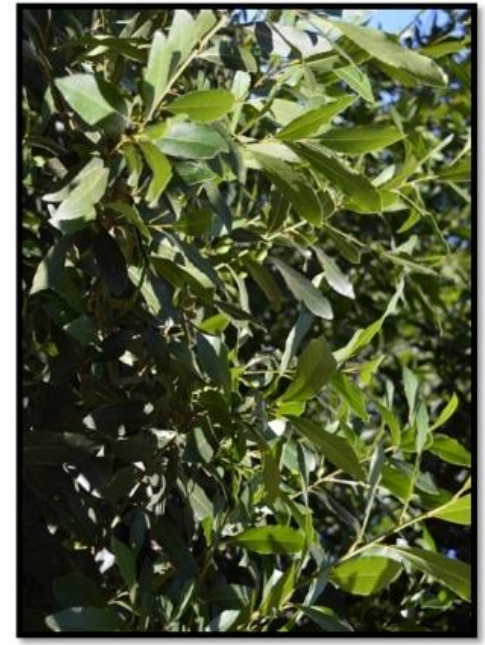

(b)

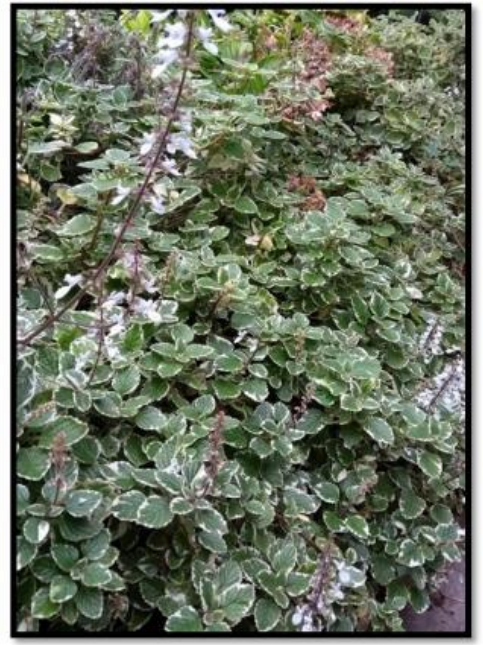

(c)

Figura 1: Especies vegetales seleccionadas a) romero (Rosmarinus officinalis), b) laurel (Laurus nobilis) y c) falso incienso (Plectranthus coleoides).

\subsection{Preparación de los extractos vegetales acuosos}

Las hojas secas se trituraron en un molinillo para café (Moulinex) hasta obtener un polvo impalpable. En un vaso de precipitados de $1000 \mathrm{~mL}$ se colocaron $20 \mathrm{~g}$ de este polvo, se adicionaron $500 \mathrm{~mL}$ de agua destilada y se realizó una extracción a $60{ }^{\circ} \mathrm{C}$ durante 1 hora, bajo agitación magnética (Figura 2). A continuación se realizó una doble filtración al vacío, utilizando papel de filtro cinta negra (Quanty JP41) primero y cinta blanca (S\&S 589/2) después. Finalmente se llevó a un volumen de $1000 \mathrm{~mL}$ con agua destilada, se envasó y se conservó en heladera a $4{ }^{\circ} \mathrm{C}$, utilizándose dentro de los 5 días de preparación.

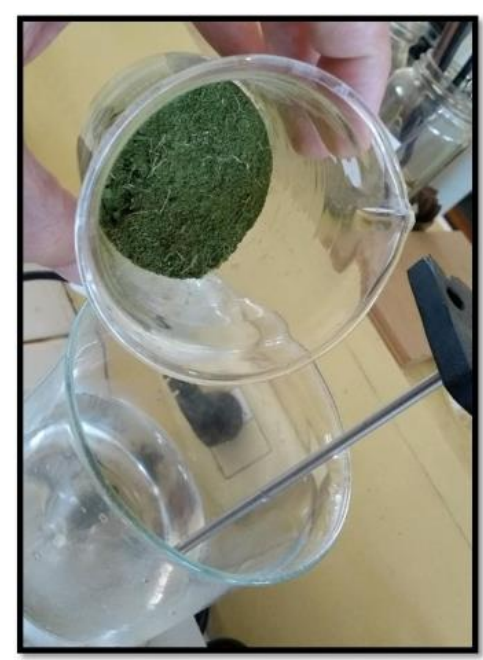

(a)

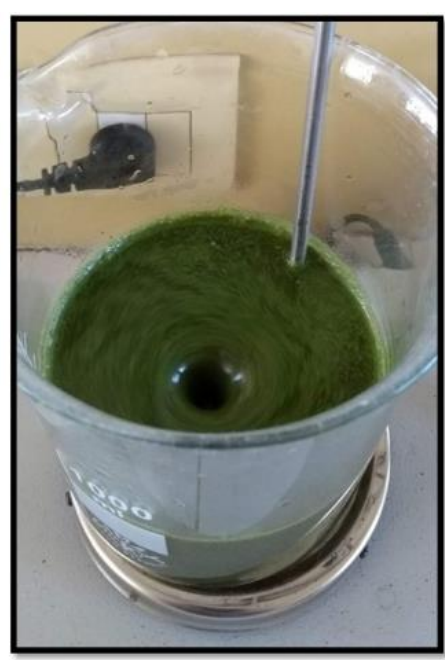

(b)

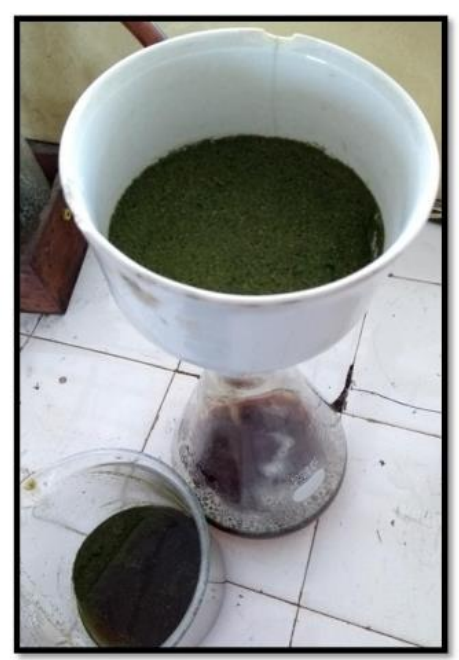

(c)

Figura 2: Preparación de los extractos vegetales acuosos por la técnica de maceración a) molienda y agregado de solvente, b) extracción con agua y c) filtración.

\subsection{Caracterización fisicoquímica de los extractos vegetales acuosos}

\subsubsection{Determinación del pH y del porcentaje de sólidos totales}

El pH de cada extracto se midió utilizando un pH-metro Arcano PHS-3E. Para determinar los sólidos totales $\mathrm{ST}$, se midieron $25 \mathrm{~mL}$ de extracto con pipeta de doble aforo y se colocó en una placa de Petri de vidrio, previamente pesada en balanza analítica Ohaus modelo Adventurer AR2140. Se secó en estufa a 95-100 ${ }^{\circ} \mathrm{C}$ hasta peso constante. Se calculó el porcentaje de sólidos totales según la Ecuación 1 (1):

$$
S T(\% p / v)=(P R-P V) * F
$$


En donde PR es el peso de la placa de Petri con residuo, PV el peso de la placa vacía y F es el factor de alícuota $(100 / 25=4)$.

En la Tabla 1 se presentan los resultados del porcentaje de los sólidos totales y el pH de los extractos acuosos. Se observa que los extractos son levemente ácidos y que la mayor acidez se registró para el extracto de laurel, seguido por el de romero y el de falso incienso, cuyos pH son muy cercanos entre sí. El análisis de los sólidos totales indica que el extracto de falso incienso es el que presenta mayor cantidad de residuo sólido, seguido por el extracto de laurel y el de romero.

Tabla 1: Porcentaje de los sólidos totales y $\mathrm{pH}$ de los extractos acuosos.

\begin{tabular}{c|c|c}
\hline EXTRACTO & PH & SÓLIDOS TOTALES (\%P/V) \\
\hline romero & 6,3 & 0,32 \\
\hline laurel & 5,7 & 0,45 \\
\hline falso incienso & 6,6 & 0,51 \\
\hline
\end{tabular}

\subsubsection{Análisis mediante espectroscopia infrarroja con transformada de Fourier (FTIR)}

Un volumen no definido de extracto se colocó en una placa de Petri y se dejó secar en estufa a $60{ }^{\circ} \mathrm{C}$. Con los sólidos obtenidos y con el pigmento ZAPP se prepararon pastillas de $\mathrm{KBr}$ y se obtuvieron los espectros FTIR correspondientes para el rango de $4000-450 \mathrm{~cm}^{-1}$ con una resolución de $4 \mathrm{~cm}^{-1}$ y 10 barridos. El espectrómetro IR utilizado fue el Spectrum One de Perkin Elmer.

\subsection{Ensayos electroquímicos}

La evaluación electroquímica de la capacidad inhibidora de los extractos se realizó a través de ensayos de polarización lineal. Para cada extracto es necesario determinar la dilución adecuada que provea una concentración óptima de inhibidores, ya que concentraciones insuficientes o bien excesivas podrían tener un efecto activador de la corrosión. Para ello se estudiaron distintas diluciones $(1 / 3,1 / 5,1 / 10,1 / 20)$ de los extractos acuosos de cada una de las tres especies vegetales en $\mathrm{NaCl} 0,1 \mathrm{M}$ sobre electrodos de acero SAE 1010 y con agitación mecánica constante a $300 \mathrm{rpm}$.

Los ensayos de polarización lineal se realizaron a las 2, 5 y 24 horas de contacto metal-solución con un potenciostato Gamry Interface 1000, seleccionando un rango de barrido de $\pm 20 \mathrm{mV}$ a partir del potencial a circuito abierto y utilizando una velocidad de barrido de $0,25 \mathrm{mV} \cdot \mathrm{s}^{-1}$. La celda utilizada estaba constituida por un electrodo de acero SAE $1010 \mathrm{de} 0,28 \mathrm{~cm}^{2}$ de área expuesta como electrodo de trabajo, un electrodo de Pt como contraelectrodo y un electrodo de calomel saturado (ECS) como electrodo de referencia. Los electrodos de trabajo fueron pulidos con lijas "al agua" (con alúmina como mineral abrasivo y con número de granos 220, 320, 400 y 600) mojadas con alcohol etílico. Luego fueron limpiados con un algodón embebido con el mismo alcohol, secados al aire $\left(5 \mathrm{~min}, 20^{\circ} \mathrm{C}\right)$ y utilizados inmediatamente previo a su empleo). La densidad de corriente de corrosión (Ic) se calculó por medio del software Gamry Echem Analyst Version 6.33. Este software permite obtener la resistencia a la polarización Rp a partir de la pendiente del gráfico corriente-potencial, y calcula Ic a través de la ecuación de Stern-Geary (2):

$$
\text { Ic }=B / A \cdot R p \quad B=(b a \cdot b c /[2,303(b a+b c)]
$$

donde ba y bc son las pendientes de Tafel anódica y catódica, respectivamente, las cuales fueron configuradas en un valor de 0,12 V/década, y A es el área expuesta.

La eficiencia inhibidora EI\% para las distintas concentraciones de extracto a los distintos tiempos se calculó según la Ecuación 3 (3):

$$
E I \%=100 \cdot\left(I_{0}-I\right) / I_{0}
$$

en donde $\mathrm{I}_{0}$ indica la Ic del blanco, esto es, una solución de $\mathrm{NaCl}$ 0,1 M sin adición de extracto, mientras que I es la Ic de la muestra correspondiente. Para cada dilución de extracto y cada tiempo de exposición las medidas se realizaron por triplicado $[3,4,6]$.

A efectos comparativos, se realizaron ensayos similares en donde en lugar del extracto se utilizó una suspensión al $1 \%$ del pigmento anticorrosivo ZAPP.

\subsection{Estudio del desempeño anticorrosivo mediante microscopía electrónica de barrido (SEM) y espectroscopia de dispersión de rayos $X$ (EDX)}

A partir de los ensayos electroquímicos, se seleccionaron para cada una de las tres especies vegetales las 
diluciones de los extractos acuosos que brindaron la mayor EI\%.

Luego, se colocaron $200 \mathrm{~mL}$ de la dilución seleccionada en un vaso de precipitados de $400 \mathrm{~mL}$ en contacto con una muestra de acero SAE 1010 durante 24 horas, con un agitador de paleta a $300 \mathrm{rpm}$. Asimismo, se realizó el ensayo "blanco" en el cual una muestra de acero SAE 1010 se pone en contacto con una solución de $\mathrm{NaCl}$ 0,1M sin adición de extracto y otro ensayo utilizando el pigmento ZAPP como referencia. La superficie metálica fue preparada de manera similar a la requerida para el estudio de polarización lineal.

Finalmente, cada una de las muestras de acero ensayadas fue enjuagada con agua destilada, secada y analizada por SEM con un equipo Philips SEM 505, mientras que la composición elemental de la superficie fue determinada por EDX con un instrumento Apex 2 EDAX modelo Apollo X.

\section{RESULTADOS}

\subsection{Caracterización fisicoquímica de los extractos vegetales acuosos}

\subsubsection{Análisis mediante espectroscopia infrarroja con transformada de Fourier (FTIR)}

Los espectros FTIR de los sólidos obtenidos para cada uno de los extractos (Figura 3), indican la presencia de grupos funcionales característicos de los fitocompuestos. Las uniones C-O en alcoholes, ésteres, éteres, y $\mathrm{C}=\mathrm{O}$ en cetonas, ésteres y ácidos carboxílicos, se pueden observar en las regiones de $1050-1300 \mathrm{~cm}^{-1}$ y 1690 $1770 \mathrm{~cm}^{-1}$ respectivamente. Por otro lado, en la región comprendida entre 2800 y $3000 \mathrm{~cm}^{-1}$ se encuentran las señales debidas a los estiramientos $\mathrm{C}-\mathrm{H}$ de grupos $\mathrm{CH}_{2} \mathrm{y} \mathrm{CH}_{3}$ [24]. Asimismo se observan unas bandas de gran intensidad en la región de 1600 y $3450 \mathrm{~cm}^{-1}$ debidas a la absorción de agua, cuya presencia está vinculada al método de extracción [25].

Para los extractos de romero y laurel se observa un hombro a $1720 \mathrm{~cm}^{-1}$ asignado al estiramiento $\mathrm{C}=\mathrm{O}$, grupo que se encuentra presente en alcanfor y acetato de terpenilo. También se encuentran señales a 1400, 1275 y $1060 \mathrm{~cm}^{-1}$ en los extractos de romero y laurel, asignadas a flexión, estiramiento simétrico y asimétrico del grupo funcional C-O-C presente en 1,8-cineol [26, 27]. En la muestra correspondiente al extracto de romero se observa además una señal a $1530 \mathrm{~cm}^{-1}$ asignada al movimiento flexión de la unión $\mathrm{C}=\mathrm{C}$, que puede asociarse al $\beta$-cariofileno.

En el extracto de falso incienso se distinguen a 705 y $810 \mathrm{~cm}^{-1}$ las vibraciones de flexión C-H fuera del plano propias de compuestos aromáticos, las cuales pueden vincularse al carvacrol [28, 29].

El espectro de la muestra ZAPP presenta, además de las bandas correspondientes a la absorción de agua, una señal entre 800 y $1100 \mathrm{~cm}^{-1}$ asociada a la presencia del grupo $\mathrm{PO}_{4}^{-3}$ y una banda en $635 \mathrm{~cm}^{-1}$ asociada a la unión P-O-P característica de los polifosfatos [30, 31].

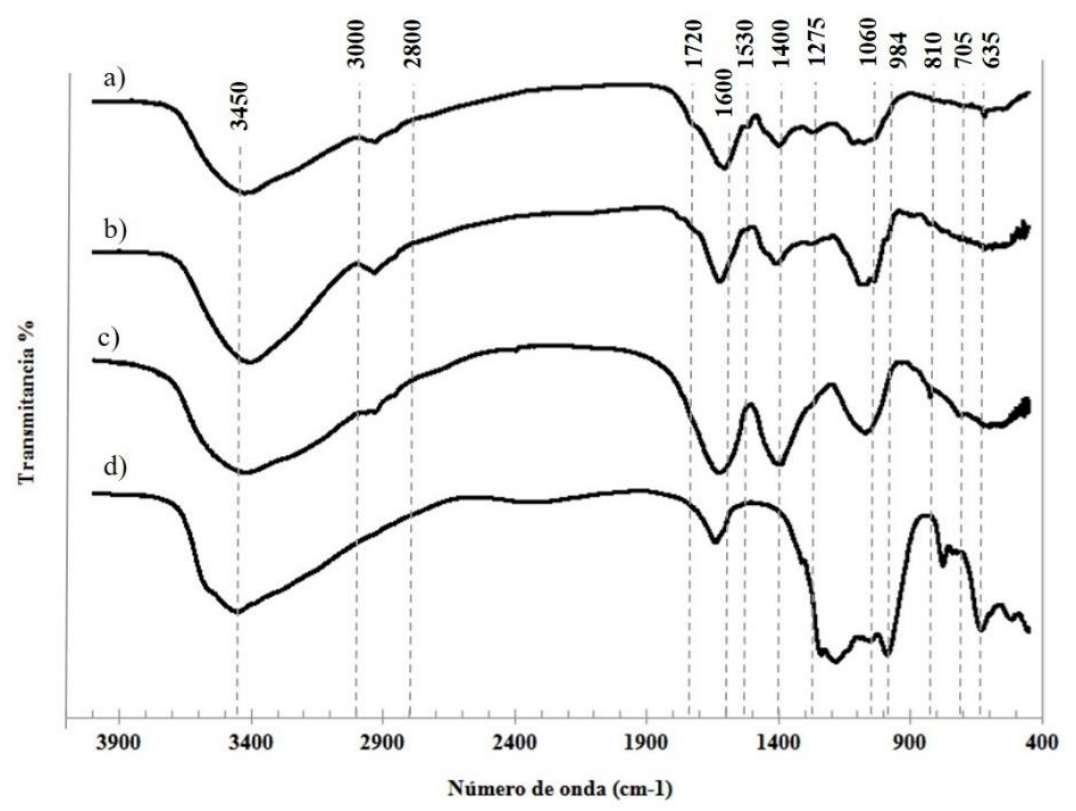

Figura 3: Espectros FTIR de los sólidos obtenidos de cada uno de los extractos a) romero, b) laurel, c) falso incienso y d) ZAPP. 


\subsection{Ensayos electroquímicos}

Los resultados de la eficiencia inhibidora porcentual para cada dilución se presentan en la Tabla 2 junto con los obtenidos para el pigmento de origen comercial ZAPP. Luego de 24 horas de inmersión, para los extractos de romero y laurel se encontró la máxima EI para la dilución 1/10, mientras que la dilución del extracto de falso incienso que mostró la mejor EI fue la 1/3. Al comparar estos resultados con los obtenidos para ZAPP se observa que las diluciones de romero y laurel presentan una eficiencia similar a la otorgada por ZAPP (86-88\%), mientras que la dilución 1/3 de falso incienso presenta aún mejores resultados (95\%).

Como se mencionó previamente, estos extractos poseen varios componentes que pueden adsorberse a la superficie metálica a través de la interacción entre los pares de electrones libres de sus heteroátomos y los electrones de sus sistemas $\pi$ con los orbitales d vacantes del hierro, dando como resultado una película protectora que enlentece el proceso de corrosión. Sin embargo, para cada extracto acuoso resulta difícil asociar la eficiencia inhibidora con un determinado componente en particular, ya que todos ellos consisten en mezclas complejas que contienen múltiples fitoquímicos con potencial acción anticorrosiva, los que además pueden tener un efecto sinérgico entre sí.

Tabla 2: Eficiencia inhibidora \% de los extractos y de ZAPP

\begin{tabular}{l|c|c|c|c|c}
\hline \multirow{2}{*}{ INHIBIDOR } & \multirow{2}{*}{ DILUCIÓN } & $\begin{array}{c}\text { CONCENTRACIÓN } \\
\text { \% P/V }\end{array}$ & \multicolumn{3}{|c}{ El \% } \\
\cline { 4 - 6 } & & 2 HORAS & 5 HORAS & 24 HORAS \\
\hline \multirow{4}{*}{ romero } & $1 / 3$ & 0,11 & 76,6 & 80,7 & 81,1 \\
\cline { 2 - 6 } & $1 / 5$ & 0,06 & 83,6 & 82,2 & 79,6 \\
\cline { 2 - 6 } & $1 / 10$ & 0,03 & 85,3 & 86,6 & 88,2 \\
\cline { 2 - 6 } & $1 / 20$ & 0,02 & 82,9 & 82,0 & 82,7 \\
\hline \multirow{4}{*}{ laurel } & $1 / 3$ & 0,15 & 76,9 & 80,9 & 83,2 \\
\cline { 2 - 6 } & $1 / 5$ & 0,09 & 82,0 & 86,4 & 84,3 \\
\cline { 2 - 6 } & $1 / 10$ & 0,05 & 52,5 & 76,8 & 86,4 \\
\cline { 2 - 6 } & $1 / 20$ & 0,02 & 50,5 & 48,2 & 64,2 \\
\cline { 2 - 6 } & $1 / 3$ & 0,17 & 84,8 & 88,2 & 95,6 \\
\cline { 2 - 6 } & $1 / 5$ & 0,10 & 78,9 & 83,6 & 89,5 \\
\cline { 2 - 6 } & $1 / 10$ & 0,05 & 74,7 & 75,8 & 85,9 \\
\cline { 2 - 6 } & $1 / 20$ & 0,03 & 60,9 & 70,4 & 79,6 \\
\hline ZAPP & ---- & 1,00 & 83,0 & 86,3 & 88,1 \\
\hline
\end{tabular}

\subsection{Estudio del desempeño anticorrosivo mediante microscopía electrónica de barrido (SEM) y espectroscopia de dispersión de rayos $\mathrm{X}$ (EDX)}

Las muestras de acero SAE 1010 sumergidas durante 24 horas en $\mathrm{NaCl}$ 0,1 $\mathrm{M}$ y en las diluciones $1 / 10$ de romero, $1 / 5$ de laurel y $1 / 3$ de falso incienso se observaron mediante SEM (Figura 4) con la finalidad de caracterizar el film obtenido. En el espectro de la muestra sin extracto y en el correspondiente al pigmento utilizado como referencia, se observa una banda debido al metalizado con oro; las demás muestras no fueron metalizadas y por ello no se observa la banda antes mencionada.

La microfotografía de acero SAE 1010 en contacto con la solución de $\mathrm{NaCl}$ 0,1 M muestra una superficie atacada por el medio agresivo, con abundante formación de productos de corrosión, mientras que en el caso del acero expuesto a las diluciones de los extractos en $\mathrm{NaCl} 0,1 \mathrm{M}$ se observa escasa formación de productos de corrosión y las líneas de pulido son claramente visibles, lo que denota la formación de películas protectoras delgadas. Para la muestra de acero en contacto con el extracto de romero se observa además, una distribución homogénea de partículas, la misma es menos evidente para la muestra en contacto con el extracto de laurel y está ausente en la muestra en contacto con el extracto de falso incienso.

En el caso de la muestra sumergida en la suspensión de ZAPP se observa la formación de un film delgado (ya que es posible observar las estrías generadas durante el pulido), en el que se destaca la presencia de $\mathrm{Zn}$ proveniente del pigmento.

La composición de las películas obtenidas, determinada mediante el análisis EDX, se presenta en la Tabla 3. 
Tabla 3: Composición de las películas mediante el análisis EDX y relación Fe/O

\begin{tabular}{l|c|c|c|c|c|c}
\hline & $\begin{array}{l}\text { OXÍGENO } \\
\% \mathbf{P} / \mathbf{P}\end{array}$ & $\begin{array}{l}\text { HIERRO } \\
\% \mathbf{P} / \mathbf{P}\end{array}$ & $\begin{array}{l}\text { CARBONO } \\
\% \mathbf{P} / \mathbf{P}\end{array}$ & $\begin{array}{l}\text { ZINC } \\
\% \mathbf{P} / \mathbf{P}\end{array}$ & TOTAL & FE/O \\
\hline blanco & 18,73 & 81,27 & ----- & ----- & 100 & 4,34 \\
\hline romero & 15,80 & 73,51 & 10,69 & ---- & 100 & 4,65 \\
\hline laurel & 9,65 & 71,55 & 18,80 & ---- & 100 & 7,41 \\
\hline falso incienso & 3,79 & 86,14 & 10,07 & ---- & 100 & 22,73 \\
\hline ZAPP & 1,26 & 97,36 & ----- & 1,38 & 100 & 77,26 \\
\hline
\end{tabular}

A partir de los datos de la Tabla 3 se observa que el film protector obtenido por el efecto de los extractos vegetales es de naturaleza orgánica, debido a la cantidad de carbono presente. Por otro lado la relación Fe/O, indicadora de la formación de productos de corrosión oxigenados, es máxima para falso incienso, seguida por laurel y romero, por lo que se puede decir que la película generada por el falso incienso otorga mayor protección que las obtenidas con los otros dos extractos. La mejor relación Fe/O, sin embargo, es la obtenida con ZAPP, aunque debe tenerse en cuenta que su concentración es dos órdenes de magnitud mayor que la de los extractos.
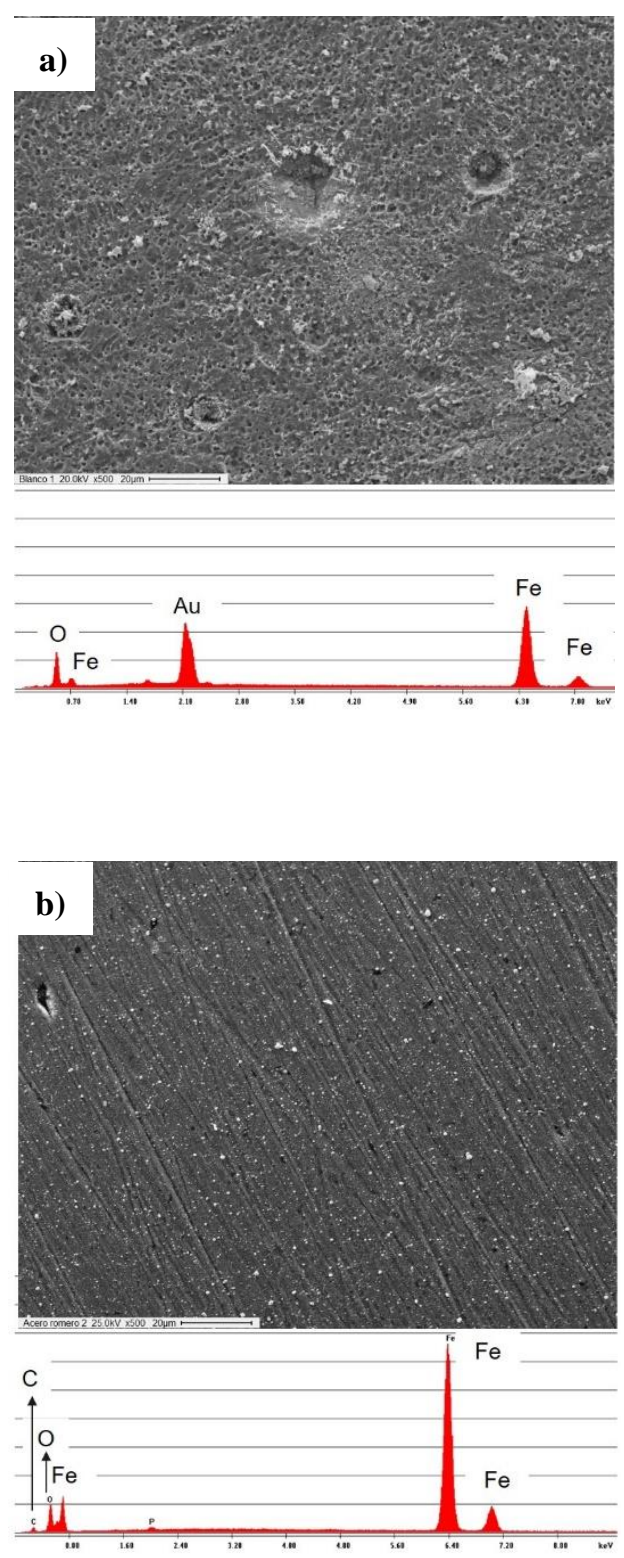

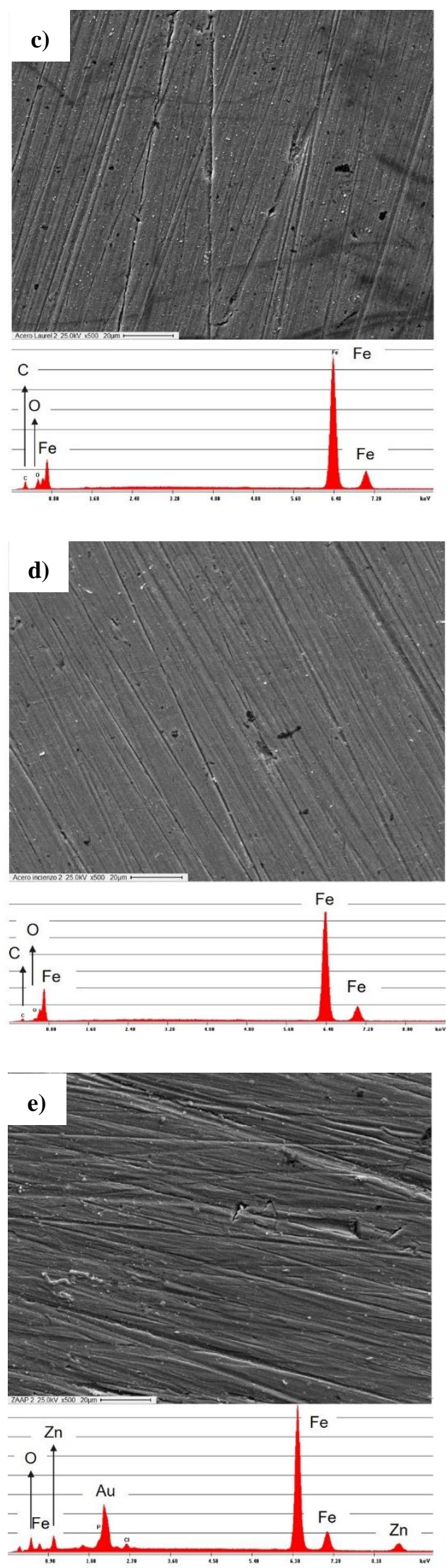

Figura 4: Microfotografías a 500x de las muestras de SAE 1010 sumergidas durante 24 h en a) $\mathrm{NaCl}$ 0,1 M, b) dilución de romero en $\mathrm{NaCl}$ 0,1M, c) dilución de laurel en $\mathrm{NaCl}$ 0,1 M, d) dilución de falso incienso en $\mathrm{NaCl}$ 0,1 $\mathrm{M}$ y e) suspensión de ZAPP en $\mathrm{NaCl}$ 0,1 M. 


\section{CONCLUSIONES}

Las principales conclusiones de este estudio se destacan a continuación:

- Mediante la técnica de maceración resultó posible la obtención de extractos acuosos de romero, laurel y falso incienso.

- La caracterización mediante FTIR indicó la presencia de grupos funcionales presentes en los componentes mayoritarios de los extractos.

- Los ensayos electroquímicos permitieron determinar la concentración óptima de los extractos inhibidores, los que a su vez presentaron una acción anticorrosiva comparable a la de un fosfato de tercera generación de origen comercial.

- Los estudios de SEM y EDX del desempeño anticorrosivo de los extractos indicaron la formación de un film protector con mejor relación $\mathrm{Fe} / \mathrm{O}$ para falso incienso, resultado que es acorde al obtenido mediante polarización lineal.

- Estos resultados indican que los extractos de romero, laurel y falso incienso inhiben la corrosión del acero SAE 1010, y podrían ser incorporados en pinturas de base acuosa como una alternativa ecológica para la protección de este metal.

\section{AGRADECIMIENTOS}

Los autores agradecen al Consejo Nacional de Investigaciones Científicas y Técnicas (CONICET), a la Universidad Nacional de La Plata (UNLP) y a la Comisión de Investigaciones Científicas de Buenos Aires (CICPBA), por el apoyo económico brindado para llevar a cabo esta investigación.

\section{BIBLIOGRAFÍA}

[1] BARUTHIO, F., "Toxic Effects of Chromium and Its Compounds", Biological Trace Element Research, v. 32, pp. 145-53, Jan. 1992.

[2] NASIR KHAN, M., MOHAMMAD, M., "Eutrhophication: Challenges and solutions". In: Ansari A., Gill S. (eds), Eutrophication: causes, consequences and control, Chapter 1, New York, USA, Springer, 2014.

[3] D’ALESSANDRO, O., SELMI, G.J., DEYÁ, C., et al.,"Formulation and Assessment of a Wash-Primer Containing Lanthanum "Tannate" for Steel Temporary Protection", Journal of Materials Engineering and Performance, v. 27, n. 2 ,pp. 687-704, Dec. 2017.

[4] D'ALESSANDRO, O., SELMI, G.J., DEYÁ, C., et al., "Lanthanum Derivative from "Tara" Tannin for Steel Temporary Protection”, Industrial \& Engineering Chemistry Research, v. 57, n. 9, pp. 3215-3226, Feb. 2018.

[5] ROSELLI, S., DEYÁ, C., REVUELTA, M., et al., "Zeolites as reservoirs for Ce(III) as passivating ions in anticorrosion paints", Corrosion Reviews, v. 36, n. 3, pp. 305-322, Jan. 2018.

[6] D'ALESSANDRO, O., SELMI, G.J., DI SARLI, A.R., et al., "Accelerated tests, a necessary complement of electrochemical assays to evaluate anti-corrosive coatings", Journal of Applied Electrochemistry, v. 49, n. 8, pp. 811- 822, Jun. 2019.

[7] RAJA, A.S., RAJENDRAN, S., NAGALAKSHMI, R., et al., "Eco-friendly inhibitor glycine- $\mathrm{Zn}^{+2}$ system controlling corrosion of carbon steel in well water", European Chemical Bulletin, v. 2, n. 3, pp. 130-136, 2013.

[8] HAMADI, L., MANSOURI, S., OULMI, K., et al., "The use of amino acids as corrosion inhibitors for metals: A review", Egyptian Journal of Petroleum, v. 27, n. 4, pp. 1157-1165, Dec. 2018.

[9] BIANCHI, A., "The Mediterranean aromatic plants and their culinary use", Natural Product Research, v. 29, n. 3, pp. 201-206, Sep. 2014.

[10] HEMALATHA, M., THIRUMALAI, T., SARANYA, R., et al., "A review on antimicrobial efficacy of some traditional medicinal plants in Tamilnadu", Journal of Acute Disease, v. 2, n. 2, pp. 99-105, Jan. 2013.

[11] BORGES, R.S., SÁNCHEZ ORTIZ, B.L., PEREIRA, A.C.M., et al., "Rosmarinus officinalis essential oil: A review of its phytochemistry, anti-inflammatory activity, and mechanisms of action involved", Journal of Ethnopharmacology, v. 229, pp. 29- 45, Jan. 2019.

[12] BOUNAAS, K., BOUZIDI, N., DAGHBOUCHE, Y., et al., "Essential oil counterfeit identification through middle infrared spectroscopy", Microchemical Journal, v. 139, pp. 347-356, Jun. 2018.

[13] AINANE, A., KHAMMOUR, F., CHARAF, S., et al., "Chemical composition and insecticidal activity of five essential oils: Cedrus atlantica, Citrus limonum, Rosmarinus officinalis, Syzygium aromaticum and Eucalyptus globules", Materials Today: Proceedings, v. 13, n. 3, pp. 474-485, Jan. 2019. 
[14] PEIXOTO, L.R., ROSALEN, P.L., FERREIRA, G.L.S., et al., "Antifungal activity, mode of action and anti-biofilm effects of Laurus nobilis Linnaeus essential oil against Candida spp.", Archives of Oral Biology, v. 73, pp. 179-185, Jan. 2017.

[15] ORDOUdI, S.A., PAPAPOSTOLOU, M., KOKKINI, S., et al., "Diagnostic Potential of FT- IR Fingerprinting in Botanical Origin Evaluation of Laurus nobilis L. Essential Oil is Supported by GC- FIDMS Data", Molecules, v. 25, n. 3, pp. 583-599, Jan. 2020.

[16] BANDEIRA, J.M., BARBOSA, F.F., BARBOSA, L.M.P., et al., "Composição do óleo essencial de quatro espécies do gênero Plectranthus", Revista Brasileira de Plantas Medicinais, v. 13, n. 2, pp. 167-164, 2011.

[17] ROJAS, L.B., CORDERO DE ROJAS, Y., CARMONA ARZOLA, J., et al., "Componentes volátiles de Plectranthus coleoides Benth. (Lamiaceae)", Revista de la Facultad de Farmacia, v. 54, n. 1, pp. 17-20, Oct. 2012.

[18] AGNANIET, H., AGREBI, A., BIKANGA, R., et al., "Essential Oil of Plectranthus tenuicaulis Leaves from Gabon, Source of (R),(E)-6,7-Epoxyocimene. An Unusual Chemical Composition within the Genus Plectranthus", Natural Product Communications, v. 6, n. 3, pp. 409-416, Mar. 2011.

[19] ROCHA J.C., GOMES, J.A.C.P.,, D’ELIA E., "Corrosion inhibition of carbon steel in hydrochloric acid solution by fruit peel aqueous extracts", Corrosion Science, v. 52, n. 7, pp. 2341-2348, Jul. 2010.

[20] VERMA, C., EBENSO, E.E., BAHADUR, I., et al., "An overview on plant extracts as environmental sustainable and green corrosion inhibitors for metals and alloys in aggressive corrosive media", Journal of Molecular Liquids, v. 266, pp. 577-590, Sep. 2018.

[21] AZMIR, J., ZAIDUL, I.S.M., RAHMAN, M.M., et al., "Techniques for extraction of bioactive compounds from plant materials: A review", Journal of Food Engineering, v. 117, n. 4, pp. 426-436, Aug. 2013.

[22] ANDRADE, J.M., FAUSTINO, C., GARCIA, C., et al. "Rosmarinus officinalis L.: an update review of its phytochemistry and biological activity", Future science AO, v. 4, n. 4, pp. 1-18, Feb. 2018.

[23] NADERI, R., ATTAR, M.M., "Cathodic disbondment of epoxy coating with zinc aluminium polyphosphate as a modified zinc phosphate anticorrosion pigment", Progress in Organic Coatings, v. 69, n. 4, pp. 392-395, Dec. 2010.

[24] HAMEED, I.H., IBRAHEAM, I.A., KADHIM, H. J., "Gas chromatography mass spectrum and fourier transform infrared spectroscopy analysis of methanolic extract of Rosmarinus oficinalis leaves", Journal of Pharmacognosy and Phytotherapy, v. 7, n. 6, pp. 90-106, Jun. 2015.

[25] MESSER, J.K., LUCIA, F.C., HELMINGER, P., "The pure rotational spectrum of water vapor- A millimeter, submillimeter, and far infrared analysis", International Journal of Infrared and Millimeter Waves, v. 4, pp. 505-539, Jul. 1983.

[26] BOUGHENDJIOUA, H., BOUGHENDJIOUA, Z., "Fourier transformed infrared spectroscopy analysis of constituents of Rosmarinus officinalis L. essential oil from Algeria", International Journal of Herbal Medicine, v. 5, n. 4, pp. 110-113, Jun. 2017.

[27] FERNÁNDEZ, N.J., DAMIANI, N., PODAZA, E.A., et al., "Laurus nobilis L. Extracts against Paenibacillus larvae: Antimicrobial activity, antioxidant capacity, hygienic behavior and colony strength", Saudi Journal of Biological Sciences, v. 26, n. 5, pp. 906- 912, Jul. 2019.

[28] MAROOSHES, M., PASSEL, V.A., "The infrared spectra of aromatic compounds: I. The out-of-plane C-H bending vibrations in the region 625- $900 \mathrm{~cm}^{-1}$, , Spectrochimica Acta, v. 7, pp. 14-24, Jan. 1955.

[29] VALDERRAMA, A.C.S., ROJAS, G.C., "Traceability of Active Compounds of Essential Oils in Antimicrobial Food Packaging Using a Chemometric Method by ATR-FTIR", American Journal of Analytical Chemistry, v. 8, n. 11, pp. 726-741, Nov. 2017.

[30] SANKARA NARAYANAN, T.S.N., "Surface pretreatment by phosphate conversion coatings-a review", Reviews on Advanced Materials Science, v. 9, n. 2, pp. 130-177, May. 2005.

[31] FERNANDES, K.S., ALVARENGA, E.A., BRANDÃO, P.R.G., et al, "Infrared-spectroscopy analysis of zinc phosphate and nickel and manganese modified zinc phosphate coatings on electrogalvanized steel", Revista Escola de Minas, v. 64, n. 1, pp. 45-49, Mar. 2011.

ORCID

Christian Byrne

https://orcid.org/0000-0003-0813-9157

Martín Ramírez

https://orcid.org/0000-0002-3594-2802 
Ezequiel Di Santo

Nicol Cristiano

Cecilia Deyá

Oriana D’Alessandro https://orcid.org/0000-0002-5729-2505

https://orcid.org/0000-0002-5358-093X

https://orcid.org/0000-0003-2853-5982

https://orcid.org/0000-0001-7096-5527 